\title{
MEMBERS OF THE COUNCIL
}

1939

OfFICERS

President

Vice Presidents

Secretary

Associate Secretaries

Treasurer

Librarian

Bulletin Editorial Committee

Transactions Editorial Committee

Colloquium Editorial Committee

Representatives on Board of Editors of the American Journal of Mathematics

\section{Ex-Presidents}

A. B. Coble

G. A. Henlund

H. L. RIETZ

Philip FrankLin

A. D. MichaL

H. E. Bray

Alonzo Church

ROBERT HENDERSON
W. B. Fire
SOLOMON LEFSCHETZ

Elected Members

To serve until December, 1939

To serve until December, 1940

W. J. Trjitzinsky

To serve until December, 1941

HermanN Weyl
G. C. Evans

C. R. Adams

R. E. LANGER

J. F. RitT

R. G. D. Richardson

W. L. Ayres

T. R. HOLLCROFT

M. H. INGRAHAM

T. M. Putnam

B. P. GILL

R. C. Archibald

A. A. Albert

TOMLINSON ForT

L. M. Graves

P. A. SMith

W. C. Graustein

Einar Hille

C. C. MAcDuffee

J. R. KLINE

M. H. STONE

J. D. TAMARKIN

T. H. Hildebrandt

J. F. RITT

R. L. WILDER

R. L. MOORE

WARREN WeAVER

G. T. WHYBURN

John von NeUMANN

J. L. SYNGE

E. J. McShane

H. P. ROBERTSON

\section{BOARD OF TRUSTEES}

1939

R. G. D. RichaRdSON
W. R. LONGLEY

G. W. Mullins 